estimate the intensity upon demand. Figures 1 and 2 show that the reporting method had its greatest effect at the terminal intensities; that is, there was a significant interaction of Methods and Intensities ( $F=15.50$, $\mathrm{df}=4 / 60, p<.01)$. This terminal effect may have been due to a reluctance of $S$ s to terminate performance until $E$ called for a report, though $S s$ were told they could call out the number corresponding to the final level and stop when the pain became too intense. The difference between the methods may also reflect the influence of E on S's performance. That is, the personal interaction between $E$ and $S$ in the one procedure may have increased the motivation of $S$ and thus delayed his pain reports.

The data obtained with the 5- and 10-point scales were compared and it was found that all pain intensities were reported sooner on the coarse scale. This difference $(\mathrm{F}=13.07, \mathrm{df}=1 / 15, \mathrm{p}<.01)$ may merely reflect the fact that a 10-point scale is more difficult to use than a 5-point scale and hence requires more time. At the present time there is insufficient data to determine precisely what influence the coarseness of the scale has on the ratings of a sensation, but these results indicate the presence of a substantial effect attributable to the scale unit size independent of the range of sensations judged.

The primary concern in this study was the comparison of two rating methods. The comparability of the pain functions yielded by the two methods suggests that the linearity of the curves previously obtained with the self-report procedure was not due to $S$ f fractionating time, because essentially linear functions were obtained even with irregular reporting times selected by $E$. The comparatively long interval between the last two scale points may represent an end spurt, or a tendency to assess reserve incorrectly. It may be, too, that for these comparatively light loads and the longer contraction times fatigue was attenuated by recovery phenomena. Both of these loads were well below the value at which internal pressure in the muscle occludes the blood supply (Humphreys \& Lind, 1963; Caldwell, 1967) so that some recovery was possible during the performance. The differences attributable to the main effects of me thods, though statistically significant, were small. Thus, there is no basis at the present time for saying that one method is better or more orderly for scaling pain other than on the basis of difficulties introduced by the active participation of $E$ in the rating procedure.

\section{REFERENCES}

BEECHER, H. K. Pain: One mystery solved Science, 1966, 151,840-841.

CALDWELL, L. S. The scaling of effort produced by strenuous isometric muscle contractions. USAMRL Report No. 749, 1967.

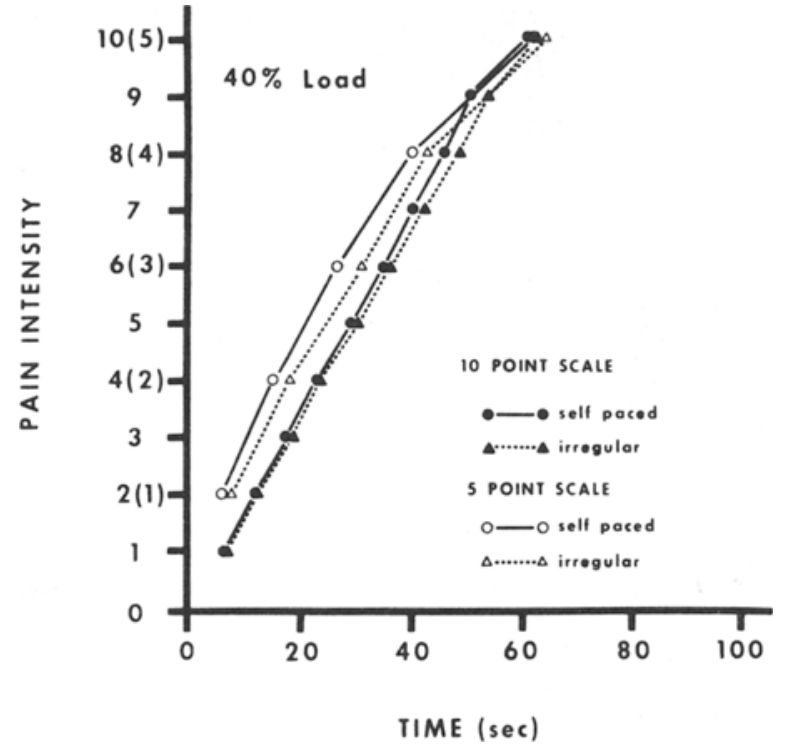

Fig. 2. Pain ratings for a $40 \%$ maximum load for both 5-point and 10-point scales using self-paced and irregular ( $E$ paced) methods of reporting.

CALDWELL, L. S., \& SMITH, R. P. Subjective estimation of effort, reserve, and ischemic pain. USAMRL Report No. 730, 1967.

HOSMAN, J. Adaptation to muscular effort. Report of the Psychological Laboratory, University of Stock holm, No. 223, 1967.

HUMPHREYS, P. W., \& LIND, A. R. The blood flow through active and inactive muscles of the forearm during sustained hand-grip contrac- tions. Journal of Physiology, 1963, 166, 120-135.

SMITH, G. M., EGBERT, L. D., MARKOWITZ, R. A., MOSTELLER, F., \& BEECHER, H. K. An experimental pain method sensitive to morphine in man: The submaximum effort tourniquet technique. Journal of Pharmacology \& Experimental Therapeutics, 1966, 154, 324-332.

\title{
Bidirectional associations in multi-trial free recall
}

RICHARD C. ANDERSON and GRAEME H. WATTS, University of Illinois, Urbana, Ill. 61801

An augmented measure of organization in free recall, combining reverse-order intertrial repetitions (R-ITR) and the usual same-order intertrial repetitions (S-ITR), was more highly correlated with measures of organization and number of words recalled than a measure including S-ITRs alone. The conditional probabilities of an S-ITR or an $R-I T R$ between one pair of trials given an $R-I T R$ between another pair of trials and the conditional probabilities of an S-ITR or an $R-I T R$ given an S-ITR were well above chance. While in every case the observed proportion of $S$-ITRs was greater than the proportion of R-ITRs, it is estimated. excluding $S s$ who alphabetized the entire list, that the majority of the observed intertrial repetitions could be explained in terms of bidirectional associations.

The intertrial repetition is a commonly used index of organization in free recall. We shall refer to the event usually investigated as a same-order intertrial repetition (S-ITR) since it involves pairs of words recalled (1) adjacent to one another, and (2) in the same order on two occasions. For example, if a recall protocol contains NECKLACE, SANDAL on two occasions, this is an S-ITR However, the appearance of NECKLACE, SANDAL on one occasion and SANDAL. NECKLACE on another is not customarily counted. The latter event is an instance of a reverse-order intertrial repetition (R-ITR). There are perfectly good theoretical reasons for including R-ITRs in the measure of organization. The R-ITR can be conceived as representing a bidirectional association and recall could be organized in part in terms of bidirectional associations. The purpose of 
Table 1

Conditional Probability of Same-Order or Trials J and J-2 given Same-Order, Reverse-Order, or No Repetition between Trials $J$ and $J-1$

\begin{tabular}{lcc}
\hline & \multicolumn{2}{c}{$\begin{array}{c}\text { Probability of repetition } \\
\text { between Trials J and J-2 }\end{array}$} \\
\cline { 2 - 3 } $\begin{array}{c}\text { Given condition } \begin{array}{c}\text { between } \\
\text { Trials J and J-1 }\end{array} \\
\text { P(S-ITR) }\end{array}$ & P(R-ITR) \\
\hline S-ITR & .330 & .181 \\
R-ITR & .158 & .224 \\
N-ITR & .077 & .058 \\
\hline
\end{tabular}

Note: The chance probability of either a same-order or reverse-order repetition is .050.

the research described in this paper was to examine the role of bidirectional associations in free recall and to determine whether there is empirical justification for an augmented measure of organization.

\section{METHOD}

Described here is a reanalysis of the data of $39 \mathrm{Ss}$ (graduate students in educational psychology) in three groups from a larger study (Watts \& Anderson, in press). Each of the three groups learned a list of 20 nouns drawn from the 1 - to 10-in-a-million frequency range (Thorndike \& Lorge, 1944). Words which formed obvious categories or associative chains were excluded from the list. The words were presented at a $1-\sec$ rate using a Lafayette No. 303A memory drum. Following each presentation, there was a $90-\mathrm{sec}$ interval for oral recall. Study and test trials alternated until a criterion of one perfect recall was reached. Then two groups received four study-test cycles on a list of 30 words capable of organization into five obvious categories. The remaining group completed arithmetic problems in place of the second list. Following the intervening activity, the Ss attempted to recall the first list. Since there were no differences between groups in speed of learning, level of organization at learning criterion, number of words recalled, from the three groups were pooled for the present analysis. Six other $S$ s in the three groups, who alphabetized the entire list on the criterion trial, were excluded from the analysis.

\section{RESULTS AND DISCUSSION}

Table 1 contains the probability of an Reverse-Order Intertrial Repetition between or level of organization at recall, the data

S-ITR or R-ITR in the comparison of the criterion trial (Trial $J$ ) with the trial two trials before (J-2), given an S-ITR, an R-ITR, or no repetition (N-ITR) between $J$ and $J-1$. Notice that the probability of a repetition given no repetition is close to the chance level whereas all of the other values are substantially above chance. These data clearly demonstrate the value of an augmented measure of organization.

The correlations between measures of organization at learning criterion ( $\mathrm{J}$ vs $\mathrm{J}-1$ ), measures of organization at recall ( $J$ vs recall trial), and number of words recalled provide further evidence for the value of an augmented measure of organization. To adjust for variations in number of words recalled, the measure employed in the correlational analysis was proportion of observed to possible repetitions. Table 2 shows that the combined measure predicts words recalled and organization at recall better than P(S-ITR) alone. Since S-ITRs and R-ITRs are mutually exclusive, it is not surprising that the correlations between $\mathrm{P}(\mathrm{S}-\mathrm{ITR})$ and P(R-ITR) based on comparisons of the same trials were low, .01 at criterion and -.16 at recall, respectively.

The following (see Bousfield \& Bousfield, 1966; Fagan, 1968) is an estimate of the proportion of same-order or reverse-order intertrial repetitions expected by chance $(\alpha)$, where $h, k$, and $c$ are, respectively, the number of words recalled on the first trial considered, the number of words recalled on the second trial considered, and the number of words common to both trials.

$$
\text { (1) } \alpha=\frac{c}{h k}
$$

The chance value for the combined measure is $2 \alpha$.

Table 3 shows that in every case the proportion of same-order repetitions was greater than the proportion of reverse-order repetitions. But this does not mean that unidirectional associations predominated since, obviously, bidirectional associations will sometimes give rise to same-order repetitions. Assume that an $S$ is in one of three states with respect to any pair of words; either he possesses a unidirectional association $(U)$, he possesses a bidirectional association (B), or he is in a residual state

Table 2

Correlations Among Proportions of Intertrial Repetitions at Learning Criterion, Proportions of Intertrial Repetitions at Recall, and Words Recalled

\begin{tabular}{lccc}
\hline & $\begin{array}{c}\text { P(S-ITR) } \\
\text { at recall }\end{array}$ & $\begin{array}{c}\text { P(S-ITR) + P(R-ITR }) \\
\text { at recall }\end{array}$ & Words recalled \\
\hline P(S-ITR) at criterion & .38 & .42 & .33 \\
P(R-ITR) at criterion & .25 & .40 & .15 \\
P(S-ITR) + P(R-ITR) at criterion & .45 & .52 & .45 \\
Words recalled & .24 & .36 & \\
\hline
\end{tabular}

Table 3

Observed Proportions of Same-Order and Reverse-Order Intertrial Repetitions

\begin{tabular}{lcc}
\hline Trials compared & P(S-ITR) & P(R-ITR) \\
\hline$J$ and J-2 & .124 & .096 \\
$\mathrm{~J}$ and $\mathrm{J}-1$ & .141 & .114 \\
$\mathrm{~J}$ and recall & .48 & .096 \\
\hline
\end{tabular}

Table 4

Estimated Proportions of Unidirectional and Bidirectional Associations Underlying Observed Distributions of Intertrial Repetitions

\begin{tabular}{lcc}
\hline Trials compared & $P(U)$ & $P(B)$ \\
\hline$J$ and J-2 & .028 & .105 \\
$\mathrm{~J}$ and J-1 & .027 & .145 \\
$\mathrm{~J}$ and recall & .052 & .108 \\
\hline
\end{tabular}

(X). Assume further that when he is in State $U$, an $S$ produces an S-ITR with probability of 1.0 , while the probability of an R-ITR or no repetition is zero. State $B$ leads to an S-ITR or an R-ITR with probability .5 , whereas, once again, the probability of an N-ITR is zero. When in State X, an S-ITR or R-ITR occurs with probability $\alpha$, while an N-ITR occurs with probability $1-2 \alpha$. The following estimates are based on these assumptions.

(2) $P(U)=P(S-I T R)-P(P-I T R)$

(3) $\mathrm{P}(\mathrm{B})=2\left[\mathrm{P}(\mathrm{R}-\mathrm{ITR})-\frac{\alpha \mathrm{P}(\mathrm{N}-\mathrm{ITR})}{1-2 \alpha}\right]$

(4) $P(X)=\frac{P(N-I T R)}{1-2 \alpha}$

Table 4 indicates that the majority of the observed intertrial repetitions can be explained in terms of bidirectional associations. However, it should be emphasized again that alphabetizers, for whom unidirectional associations would predominate, were excluded from the analysis. Notice that unidirectional associations account for a relatively greater proportion of the repetitions observed between Trial $J$ and the recall trial, suggesting that unidirectional associations are more stable than bidirectional ones.

\section{REFERENCFS}

BOUSFIELD, A. K., \& BOUSIIELD, W. A Measurement of clustering and of sequentia constancies in repeated free recall. Psychological Reports, 1966, 19, 935-942.

I AGAN, J. I: Measuring verbal recall. II: The ITR score expressed as a ratio. Psychonomic Science. $1968,11,205$.

THORNDIKE, E. L., \& LORGE. I. The teacher's word book of 30,000 words. New York Teachers College, Columbia University, 1944. WATTS, G. H., \& ANDERSON, R. C. Retroactive inhibition in free recall as a function of first and second list organization. Journal of Experimental Psychology, in press. NOTE:

1. The authors are grateful to Richard J. Bogart for his helpful suggestions. 